\title{
Platelet Rich Fibrin in Periodontal Practice- A Review
}

\author{
Subashri.A ${ }^{1}$ and Dhanraj Ganapathy ${ }^{2}$ \\ ${ }^{1}$ Saveetha Dental College and Hospitals, Saveetha Institute of Medical \\ and Technical Sciences, Saveetha University, Chennai, India \\ ${ }^{2}$ Professor and Head ,Department of Prosthodontics, Saveetha Dental College and Hospitals, \\ Saveetha Institute of Medical and Technical Sciences, Saveetha University, Chennai, India
}

\section{ABSTRACT}

Autologous platelet concentrates have become a rage in the field of regenerative medicine encompassing numerous specialties like orthopedics, maxillofacial and cosmetic surgery, sports medicine and dentistry. The use of these platelet concentrates ranging from fibrin glues to the currently used platelet-rich fibrin (PRF) has exponentially increased and it has brought about a radical change in the stimulation and acceleration of regenerative tissue processes. This review highlights the use of platelet rich fibrin in the field of periodontics and tissue repair. It brings to light the various applications of PRF procured from in vitro, animal and human clinical trials

\section{KEY WORDS: PLATELET RICH CONCENTRATES; PLATELET RICH FIBRIN; PERIODONTICS; REGENERATION; TISSUE} REPAIR.

\section{INTRODUCTION}

The activated form of a plasmatic molecule called fibrinogen is known as fibrin. This soluble fibrillary molecule is massively present both in plasma and in the platelet granules and plays a pivotal role in platelet aggregation during haemostasis. The biochemical process involves the transformation of it into a biologic glue capable of consolidating the initial platelet cluster, thus constituting a protective wall along vascular breaches during coagulation. Fibrinogen remains the final substrate in all coagulation reactions. Fibrinogen is a soluble protein and it gets transformed into insoluble fibrin by the enzyme known as thrombin. The resultant polymerized fibrin gel constitutes the first cicatricial

\section{ARTICLE INFORMATION}

*Corresponding Author: dhanraj@saveetha.com

Received 16th June 2020 Accepted after revision 5th August 2020 Print ISSN: 0974-6455 Online ISSN: 2321-4007 CODEN: BBRCBA

Thomson Reuters ISI Web of Science Clarivate Analytics USA and Crossref Indexed Journal

\section{Clarivate
Analytics}

NAAS Journal Score 2020 (4.31) SJIF: 2020 (7.728)

A Society of Science and Nature Publication,

Bhopal India 2020. All rights reserved.

Online Contents Available at: http//www.bbrc.in/

Doi: http://dx.doi.org/10.21786/bbrc/13.7/82 matrix of the injured site. Fibrin adhesives, even though controversial, were commonly used due to its healing properties i.e., potential to accelerate healing and reduce the formation of postoperative hematoma.

Platelets contribute to homeostasis by preventing blood loss at sites of vascular injury, and they contain a large number of growth factors and cytokines that have a key role in bone regeneration and soft-tissue maturation. In the past years, an improved understanding of the physiological role of platelets in the wound healing and after tissue injury has led to the idea of using platelets as therapeutic tools. Indeed, after fibrin glue was introduced in the early 1990s as a biomaterial with haemostatic and adhesive properties, the strategic modification of the fibrin to include platelets was reported. The source of the new preparation, known as platelet-rich plasma (PRP), consists of a limited volume of plasma enriched in platelets, which is obtained from the patient.

Once the platelet concentrate is activated by way of thrombin generation with calcium, a three-dimensional and bio compatible fibrin scaffold is formed, and a myriad of growth factors and proteins are released, progressively,

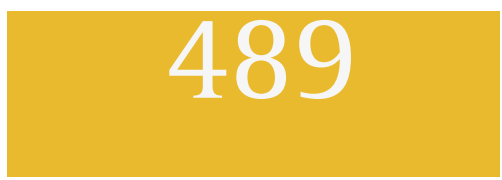


to the local environment, contributing to the accelerated postoperative wound healing and tissue repair.

Previously our department has published extensive research on various aspects of prosthetic dentistry (Anbu et al., 2019; Ariga et al., 2018; Ashok and Ganapathy, 2019; Duraisamy et al., 2019; Ganapathy et al., 2017; Gupta et al., 2018; Jain, 2017a, 2017b; Ranganathan et al., 2017; Varghese et al., 2019), this vast research experience has inspired us to review platelet rich fibrin in periodontal practice.

Preparation of Prf for Clinical Use: Platelets are formed by a peripheral area, which corresponds to a phospholipid membrane, a series of microtubules, and a very extensive canalicular system connecting the surface to the cytoplasm. Glycogen granules, mitochondria, lysosomes, peroxisomes, and various types of inclusions, including alpha and dense granules, can be identified within the cytoplasm.

Blood samples from non-smoking, healthy male donors and PRF clots are collected and prepared immediately or after storage for 1-2 days. Fibrin fibers are examined by scanning electron microscopy. Bioactivity was assessed by means of a bioassay system involving the human periosteal cells, whereas PDGF-BB concentrations were determined using an enzyme-linked immunosorbent assay. PRF is prepared by a process known as differential centrifugation. In differential centrifugation, acceleration force is adjusted to sediment certain cellular constituents based on different specific gravity(Harris and Hillyer, 2007). There are many ways of preparing PRF. It can be prepared by the PRF method or by the buffy-coat method(Man et al., 2001).

Clinical Use of Prf in Periodontics Practice: PRF comprises of an autologous leukocyte-platelet-rich fibrin matrix (Choukroun et al., 2006a) composed of a tetra molecular structure, with cytokines, platelets, cytokines, and stem cells within it, which acts as a biodegradable scaffold (Li et al., 2013)that favors the development of microvascularization and is able to guide epithelial cell migration to its surface (Chang and Zhao, 2011). In surgical interventions, PRF could serve as a resorbable membrane for guided bone regeneration (GBR), preventing the migration of non-desirable cells into bone defect and providing a space that allows the immigration of osteogenic and angiogenic cells and permits the underlying blood clot to mineralize.PRF may act as a biologic adhesive to hold the particles together, facilitating the manipulation of the bone grafts.

PRF membrane has exhibited favorable clinical results in the treatment of periodontal infrabony defects (Chang and Zhao, 2011), protecting open wounds from oral environment when the suture cannot bind the mucosal margins (Simonpieri et al., 2012), and accelerating hard and soft tissue healing. Some clinical studies used PRF membrane as a sole grafting material to achieve maxillary sinus floor augmentation, presenting promising results.
Choukroun et al. (Choukroun et al., 2006b) conducted a study in which they wanted to see the potential of using PRF in conjunction with freeze-dried bone allograft with (FDBA) to enhance bone regeneration in a maxillary sinus lift procedure. The results showed a decreased healing time prior to implant placement. It can be utilized in various procedures such as management of intrabony defects, gingival recession, furcation defects, extraction socket preservation, and accelerated healing of wounds.

In Vitro Studies: PRF is enriched with platelets, growth factors and cytokines increasing the healing potential of both hard and soft tissue(Hotter, 1984). There are only scant references in the literature about the biologic properties of PRF when compared to the other platelet concentrates. The literature predominantly contains animal and human studies of the experimental use of PRF and only limited in vitro studies have been carried out on the effects of PRF on cell proliferation. Despite the lack of scientifically proven clinical benefits, PRF is considered as a healing biomaterial and is commonly used in implant and plastic periodontal surgery procedures to enhance bone regeneration and soft-tissue wound healing (Dohan et al., 2006). According to Choukron et al. PRF was initially used in implant surgery to enhance the healing properties of the bone(Miron and Choukroun, 2017).

Animal Studies: PRF is a highly complex architecture of strong fibrin matrix as a resorbable membrane with growth factors released after at least 1 week. Therefore, PRF can act as a reservoir of many growth factors that are known to play a crucial role in hard and soft tissue repair processes. Previously, it has been reported that PRF has a strong positive effect on the pro- liferation of gingival fibroblasts, periodontal ligament fi- broblasts, and osteoblasts, but retards epithelial cell proliferation in vitro.This cell-type-specific manner makes PRF beneficial to periodontal regeneration. Additionally, activation of phosphorylated extracellular signalregulated protein kinase, osteoprotegerin, and alkaline phosphatase expression by PRF suggests the pivots for new periodontal attachment formation.Furthermore, antimi- crobial effects of PRF have also been described. The regeneration potential capacity and antibacterial effects of PRF may be useful in periodontal surgery.

\section{DISCUSSION}

The regeneration of the lost periodontal structures is the ultimate aim of the periodontal therapy to restore the health function and esthetic of periodontium.The breakthrough in vitro study that introduced PRF in medical field a conducted by Choukroun et al (Choukroun et al., 2006a) highlighted improved neovascularization, wound closing with accelerated tissue remodeling in the absence of infectious events.

Some advantages are reported in the literature related to the use of PRF are its preparation is a simplified and efficient technique, with centrifugation in a single step, free and openly accessible for all clinicians.It 
is obtained by autologous blood sample. Minimized blood manipulation.It does not require the addition of external thrombin because polymerization is a completely natural process, without any risk of suffering from an immunological reactionIt has a natural fibrin framework with growth factors within that may keep their activity for a relatively longer period and stimulate tissue regeneration effectively.It can be used solely or in combination with bone grafts, depending on the purpose. Increases the healing rate of the grafted bone.It is an economical and quick option compared with recombinant growth factors when used in conjunction with bone grafts. When used as a membrane, it averts a donor site surgical procedure and results in a reduction in patient discomfort during the early wound-healing period.

PRF may present some disadvantages like the final amount available is low because it is autologous blood. The success of the PRF protocol depends directly on the handling, mainly, related to blood collection time and its transference for the centrifuge. Need of using a glass-coated tube to achieve clot polymerization. Possible refusal of treatment by the puncture required for blood collection.Only needs a minimal experience of clinician for PRF manipulation.

\section{CONCLUSION}

Though PRF belongs to a new generation of platelet concentrates, the biological fuction of fibrin molecules is enough in itself to account for significant cicatricial capacity of the PRF. The slow polymerization mode confers a PRF membrane as a particularly favorable physiologic architecture to support the healing process. However, it is now necessary to look further into platelet and inflammatory features of this biomaterial. Only a perfect understanding of its components and their significance will enable us to comprehend the clinical results obtained and subsequently extend the fields of therapeutic application of this protocol.

Author Contributions: Author 1 (Subashri.A) carried out retrospective study by collecting data and drafted the manuscript after performing the necessary statistical analysis. Author 2 (Dr.Dhanraj) aided in the conception of the topic, participated in the study design, statistical analysis and supervised in preparation of the manuscript and helped in study design and coordinated in developing the manuscript. All the authors have equally contributed in developing the manuscript.

\section{ACKNOWLEDGMENTS}

The authors of the study would like to acknowledge the support rendered by the Department of Prosthodontics, Media Records Department of Saveetha Dental College and Hospitals and the management for their constant assistance with the research.

Conflict of Interest: There are no conflicts of interest.

\section{REFERENCES}

Anbu RT, Suresh V, Gounder R, et al. (2019) Comparison of the Efficacy of Three Different Bone Regeneration Materials: An Animal Study. European journal of dentistry 13(1): 22-28.

Ariga P, Nallaswamy D, Jain AR, et al. (2018) Determination of Correlation of Width of Maxillary Anterior Teeth using Extraoral and Intraoral Factors in Indian Population: A Systematic Review. World Journal of Dentistry 9(1): 68-75.

Ashok V and Ganapathy D (2019) A geometrical method to classify face forms. Journal of oral biology and craniofacial research 9(3): 232-235.

Chang Y-C and Zhao J-H (2011) Effects of platelet-rich fibrin on human periodontal ligament fibroblasts and application for periodontal infrabony defects. Australian dental journal 56(4): 365-371.

Choukroun J, Diss A, Simonpieri A, et al. (2006a) Platelet-rich fibrin (PRF): A second-generation platelet concentrate. Part IV: Clinical effects on tissue healing. Oral Surgery, Oral Medicine, Oral Pathology, Oral Radiology, and Endodontology. DOI: 10.1016/j. tripleo.2005.07.011.

Choukroun J, Diss A, Simonpieri A, et al. (2006b) Platelet-rich fibrin (PRF): A second-generation platelet concentrate. Part V: Histologic evaluations of PRF effects on bone allograft maturation in sinus lift. Oral Surgery, Oral Medicine, Oral Pathology, Oral Radiology, and Endodontology. DOI: 10.1016/j.tripleo.2005.07.012.

Dohan DM, Choukroun J, Diss A, et al. (2006) Platelet-rich fibrin (PRF): a second-generation platelet concentrate. Part I: technological concepts and evolution. Oral surgery, oral medicine, oral pathology, oral radiology, and endodontics 101(3): e37-44.

Duraisamy R, Krishnan CS, Ramasubramanian H, et al. (2019) Compatibility of Nonoriginal Abutments With Implants: Evaluation of Microgap at the ImplantAbutment Interface, With Original and Nonoriginal Abutments. Implant dentistry 28(3): 289-295.

Ganapathy DM, Kannan A and Venugopalan S (2017) Effect of Coated Surfaces influencing Screw Loosening in Implants: A Systematic Review and Meta-analysis. World Journal of Dentistry 8(6): 496-502.

Gupta P, Ariga P and Deogade SC (2018) Effect of Monopoly-coating Agent on the Surface Roughness of a Tissue Conditioner Subjected to Cleansing and Disinfection: A Contact Profilometric Study. Contemporary clinical dentistry 9(Suppl 1): S122S126.

Harris SB and Hillyer CD (2007) Blood Manufacturing: Component Preparation, Storage, and Transportation. Blood Banking and Transfusion Medicine. DOI: 10.1016/ b978-0-443-06981-9.50017-x.

Hotter A (1984) The Physiology and Clinical Implications of Wound Healing. Plastic Surgical Nursing. DOI: 10.1097/00006527-198400410-00002.

Jain AR (2017a) Clinical and Functional Outcomes of 
Implant Prostheses in Fibula Free Flaps. World Journal of Dentistry 8(3): 171-176.

Jain AR (2017b) Prevalence of Partial Edentulousness and Treatment needs in Rural Population of South India. World Journal of Dentistry 8(3): 213-217.

Li Q, Pan S, Dangaria SJ, et al. (2013) Plateletrich fibrin promotes periodontal regeneration and enhances alveolar bone augmentation. BioMed research international 2013: 638043.

Man D, Plosker H and Winland-Brown JE (2001) The Use of Autologous Platelet-Rich Plasma (Platelet Gel) and Autologous Platelet-Poor Plasma (Fibrin Glue) in Cosmetic Surgery. Plastic and Reconstructive Surgery. DOI: 10.1097/00006534-200101000-00038.

Miron RJ and Choukroun J (2017) Platelet Rich Fibrin in Regenerative Dentistry: Biological Background and Clinical Indications. John Wiley \& Sons.

Ranganathan H, Ganapathy DM and Jain AR (2017)
Cervical and Incisal Marginal Discrepancy in Ceramic Laminate Veneering Materials: A SEM Analysis. Contemporary clinical dentistry 8(2): 272-278.

Simonpieri A, Del Corso M, Vervelle A, et al. (2012) Current knowledge and perspectives for the use of platelet-rich plasma (PRP) and platelet-rich fibrin (PRF) in oral and maxillofacial surgery part 2: Bone graft, implant and reconstructive surgery. Current pharmaceutical biotechnology 13(7): 1231-1256.

Varghese SS, Ramesh A and Veeraiyan DN (2019) Blended Module-Based Teaching in Biostatistics and Research Methodology: A Retrospective Study with Postgraduate Dental Students. Journal of dental education 83(4): 445-450.

World Journal of Dentistry (2017) Evaluation of Corrosive Behavior of Four Nickel-chromium Alloys in Artificial Saliva by Cyclic Polarization Test:An in vitro Study. 8(6): 477-482. 